\title{
Miniaturization of Mobile GPR Antenna Assembly
}

\author{
Di Shi ${ }^{1}$, Albert Nadal Zaragoza ${ }^{2}$, Taimur Aftab ${ }^{1}$, Gunnar Gidion ${ }^{1}$, Leonhard Reindl ${ }^{1}$ \\ ${ }^{1}$ University of Freiburg, Georges-Köhler-Allee 106, 79110 Freiburg, Germany, \\ 2 Polytechnic University of Catalonia, Carrer de Jordi Girona, 1-3. 08034 Barcelona, Spain \\ di.shi@imtek.uni-freiburg.de
}

\begin{abstract}
Summary:
For miniaturizing a mobile ground penetrating radar (GPR) antenna assembly, an electromagnetic band gap (EBG) structure to reduce the transmitting and receiving cross-talk is proposed. The antenna assembly works around $2.45 \mathrm{GHz}$ and supposed to detect motions though concrete and other building materials. Dimension of the required EBG-structure for both air-coupled and ground-coupled scenarios are analytically estimated. Finite element method based radio frequency simulations are conducted to verify the estimations and to find the optimal dimensions.
\end{abstract}

Keywords: GPR, planar antenna, miniaturization, RF-cross-talk isolation, electromagnetic band gap

\section{Introduction}

A ground penetrating radar (GPR) is capable of detecting motion even through layers of material. After an earthquake, a GPR can be used to find survivors that trapped under collapsed buildings by detecting the body motion caused by their respiration. The most volume consuming part of a traditional GPR is the transmitting and receiving ( $T x-$ and $R x-)$ antenna assembly. Beside the physical size of the antenna itself, the Tx- and Rx-antennas usually need to be spatially separated by a certain distance to reduce the cross-talk which would otherwise obliterate the signals of interest. However, increasing the Tx- and Rx antennas distance will increase the volume of the assembly, which impacts the mobility of the system.

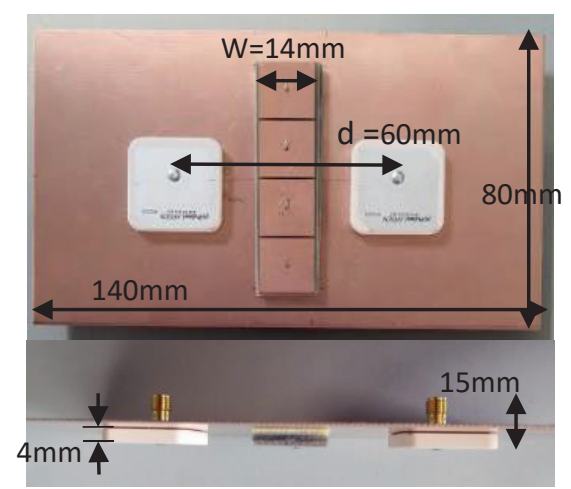

Fig. 1. TX- and Rx-antenna assembly with a 4element EBG structure, $W=14 \mathrm{~mm}$. Top: front view. Bottom: side view.

For a coplanar antenna assembly, the crosstalk occurs as surface waves between the Txand Rx antenna. In studies of metamaterial, it was found that some structures exhibit band- stop features for surface electromagnetic waves, this kind of structure is called electromagnetic band gap (EBG). There are various kinds of EBG structures, among them the mushroom-like EBG earned increasing attention in the recent decade, as first published in [2]. The mushroom-like EBG consists of evenly distributed metallic patches of the same shape, a ground plane in parallel and conducting vias (see Fig. 1. and 2). This special metallic structure introduces an LC resonator and the capacitance and inductance are determined as [2]:

$$
\begin{aligned}
& L=\mu_{0} \cdot h, \quad(1) \\
& C=\frac{W \varepsilon_{0}\left(1+\varepsilon_{r}\right)}{\pi} \cosh ^{-1}\left(\frac{2 W+s}{s}\right),
\end{aligned}
$$

in which $h$ is the EBG substrate height, $W$ is width of one EBG element, $s$ is the gap between elements and $\varepsilon_{r}$ is the relative permittivity of the substrate.

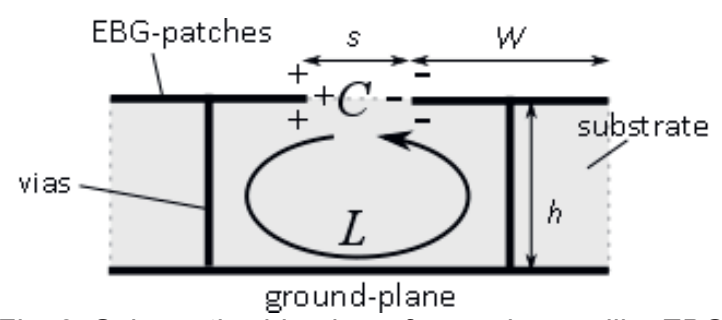

Fig. 2. Schematic side-view of a mushroom-like EBG structure with two elements. [2]

By cascading these elements, a high-order band-stop filter can be built. In [3], simulations show that the EBG provides better isolation than other surface wave reduction approaches, such as substrate removal or cavity-backed structure. 


\section{Method}

Here we work with two commercial $2.45 \mathrm{GHz}$ ceramic patch antennas [1] and restrict the analysis on single row mushroom-like square shape EBG structure (see Fig. 1). The EBG substrate is FR4 with the same height as the antenna: $4 \mathrm{~mm}$, gap between elements is 0.5 $\mathrm{mm}$. The Tx- and Rx-antennas share a $0.8 \mathrm{~mm}$ thick ground plane with a width of $80 \mathrm{~mm}$ and a length of $140 \mathrm{~mm}$. Center to center distance of the two antennas is $60 \mathrm{~mm}$. By using the resonance frequency of a simple LC-circuit:

$$
f_{\text {stop }}=\frac{1}{2 \pi \sqrt{L C}} \text {, }
$$

together with eq. (1) and (2), the required width of EBG-element $W$ can be estimated. The desired $f_{\text {stop }}$ is $2.45 \mathrm{GHz}$. For the air-coupled case, the required capacitance is about $0.84 \mathrm{pF}$ and the $W$ should be about $12 \mathrm{~mm}$. For groundcoupled case, the permittivity of ground $\varepsilon_{r, \text { grd }}$ should be considered in the equation for capacitance as:

$$
C=\frac{W \varepsilon_{0} \varepsilon_{r, g r d}\left(1+\varepsilon_{r}\right)}{\pi} \cosh ^{-1}\left(\frac{2 W+s}{s}\right)
$$

The relative permittivity of common building material is between 2 and 9 [4]. For $\varepsilon_{r, \text { grd }}=2,4$ and 6 , the required $W$ shall be 7 $\mathrm{mm}, 4 \mathrm{~mm}$ and $3 \mathrm{~mm}$ respectively.

To verify the estimation, the model is analyzed with FEM based RF simulation. For air-coupled case, the number of EBG-elements is kept as 4, $W$ varies from $10 \mathrm{~mm}$ to $18 \mathrm{~mm}$. For groundcoupled cases, the number of elements is kept as $8, W$ varies from $2 \mathrm{~mm}$ to $9 \mathrm{~mm}$.

\section{Results}

The transmission coefficient $S_{21}$ indicates the mutual coupling between Tx- and Rx- antenna.

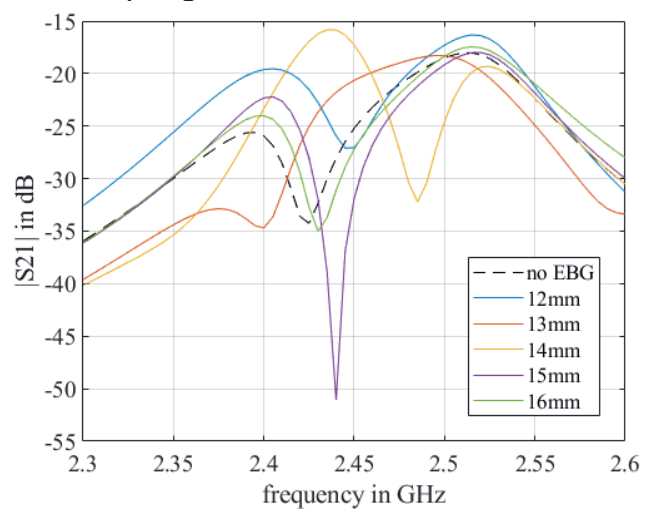

Fig. 3. $S_{21}$ of air-coupled simulation. Comparison between no EBG and a 4-element EBG of various widths.
From the air-coupled simulation (see Fig. 3), that only EBG structure with a width of $15 \mathrm{~mm}$ forms a stopband around $2.45 \mathrm{GHz}$. In the measurement, the antenna assembly with 14 $\mathrm{mm}$ EBG has the weakest mutual coupling at $2.45 \mathrm{GHz}$. From the ground-coupled simulation with $\varepsilon_{r, g r d}=4$ (see Fig. 4), $8 \mathrm{~mm}$ width EBG is most suitable to isolate the cross-talk around $2.45 \mathrm{GHz}$. However, in both scenarios the band-stop frequency is very sensitive to the EBG width, only $1 \mathrm{~mm}$ difference could lead to completely different mutual coupling in the desired frequency band.

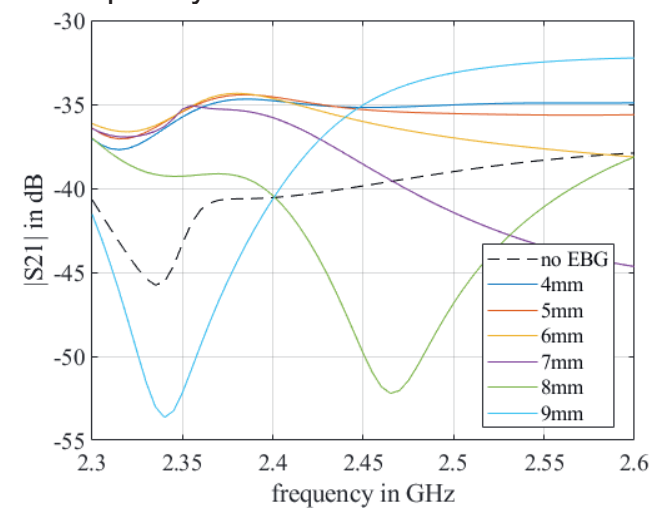

Fig. 4. $S_{21}$ of ground-coupled $\varepsilon_{r, \text { grd }}=4$ simulation,. Comparison between no EBG and an 8-element EBG of various widths.

\section{Conclusion}

In this work, a single-row EBG structure is implemented to reduce the cross-talk between planar Tx- and Rx- antennas. Simulations and measurements exhibit that the implemented EBG could reduce antenna mutual coupling by up to $10 \mathrm{~dB}$.

\section{Acknowledgment}

This research is part of the German research project SORTIE, and is supported by the Federal Ministry of Education and Research.

\section{References}

[1] $2.45 \mathrm{GHz}$ Pulse Lasern antenna W3229, https://www.pulselarsenantennas.com/

[2] D. Sievenpiper, High-impedance electromagnetic surfaces, Ph.D. dissertation, Dept. Elect. Eng., Univ. California at Los Angeles, CA, 1999.

[3] F. Yang, Y. Rahmat-Samii, Microstrip antennas integrated with electromagnetic band-gap (EBG) structures: a low mutual coupling design for array applications, IEEE Transactions on Antennas and Propagation, Oct. 2003, doi: 10.1109/TAP.2003.817983.

[4] Robert, A. Dielectric permittivity of concrete between $50 \mathrm{Mhz}$ and $1 \mathrm{GHz}$ and GPR measurements for building materials evaluation. J. Appl. Geophys. 1998, 40, 89-94, doi:10.1016/S09269851(98)00009-3. 